\title{
Importance of Capillary Hysteresis on Coreflood Experiments
}

\author{
Guo Y.
}

IBM - EPAC , Norway

\begin{abstract}
Coprright 1985, Steering Committee of the European IOR - Symposium.
This paper was presented at the 8th. European IOR - Symposium in Vienna, Austria, May 16 - 17, 1995

This paper was selected for presentation by the Steering Committee, following review of information contained in an abstract submitted by the author(s). The paper, as presented has not been reviewed by the Steering Committee.
\end{abstract}

\begin{abstract}
Coreflood experiments for obtaining relative permeability curves are often subjected to capillary effect which is neglected in the laboratory data interpretation. Due to the well-known capillary end-effect. the water saturation distribution prior to start of a flooding experiment is ofien non-uniform. In addition. the saturation at different locauion of the core has experienced different saturation history. which might make hysteresis effect signiricant in the final relative permeability cures.
\end{abstract}

This work is based on numerical simulations performed with a core flood simulator where realistic relative permeability and capillary pressure curves for both drainage and imbibition have been used. Commonly appiied coreflood process has been subjected for simulation studies. and the hysteresis effect is shown to be significant specially for the mixed-wet case. The results indicate that precautions should be paid when planning coreflood experiments.

\section{Introduction}

Coreflood experiments for obtaining relative permeability curves are often performed under the following assumptions: 1) Linear ID flow: 2) Incompressible fluids: 3) Uniform initial water saturation: 4) Negligible capillary effect. Assumption 1 and 2 are generally acceptable. However. the latter two are often violated. Capillary effect can be high around residual saturauons which are normally the initial or final conditions for a coreflood experiment. In addition. depending on various methods for establishing the initial saturation. the saturation history can be different at different locations of the core. Following the commonly accepted hysteresis theory. the relative permeability as well capillary pressure may have different values depending on the saturation history.

\section{$S_{w i}$ establishment for waterflood}

Normally. a watertlood experiment starts with establishing initial saturation to the level similar to the connate water saturauon in the reservoir. Three commonly used methods are: 1) Diaphragm drainage: 2) High rate viscous oil flooding: 3) Centrifuge drainage. Diaphragm method is the most suitable method in respect of the final saturation which can be sufficiently low and is believed uniform. However. the conventional diaphragm drainage with ceramic porous plate might take days or weeks to reach the desired saturation. Newer technique : $=$ using micropore membranes instead of ceramic ones makes the method far more attractive. Centrifuge is a good alternative considering the shor time needed to complete the operation. The largest disadvantages are the possibility for mechanical damage of core and the limitation o core lengths. High rate high viscous oilflooding is a convenient and often adapted method to establish initial water saturation prior to a watertlood. It is known that the water saturation profile at the end of oilflooding is affected by capillary pressure. In addition. hysteresis effect might be significant when the non-uniform initial water saturatuon profile experiences a redistribution before start of water injection. The latter effect has not been paid enough attention.

\section{Simulation with hysteresis option}

The coreflood simulator CENDRA ${ }^{3}$ has been used to simulate waterflood processes. CENDRA is a two-phase 2D black-oil simulator tailor-made for simulating experiments related to core analysis. A hysteresis option is available. A complete description of the hysteresis model is given in Appendix A. 

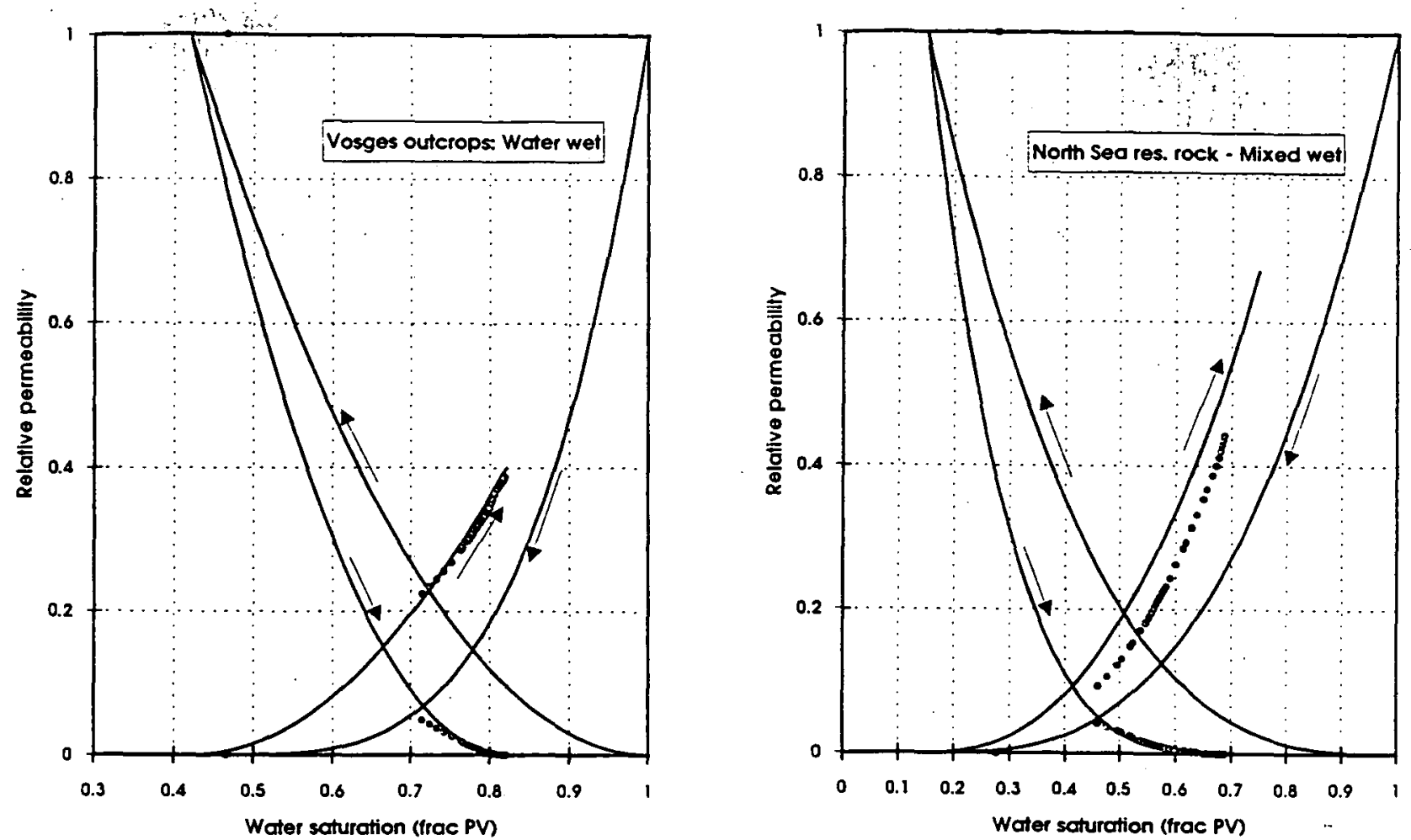

Figure 1 - Relative permeability curves: a) Water wet core; b) Mired wet core.
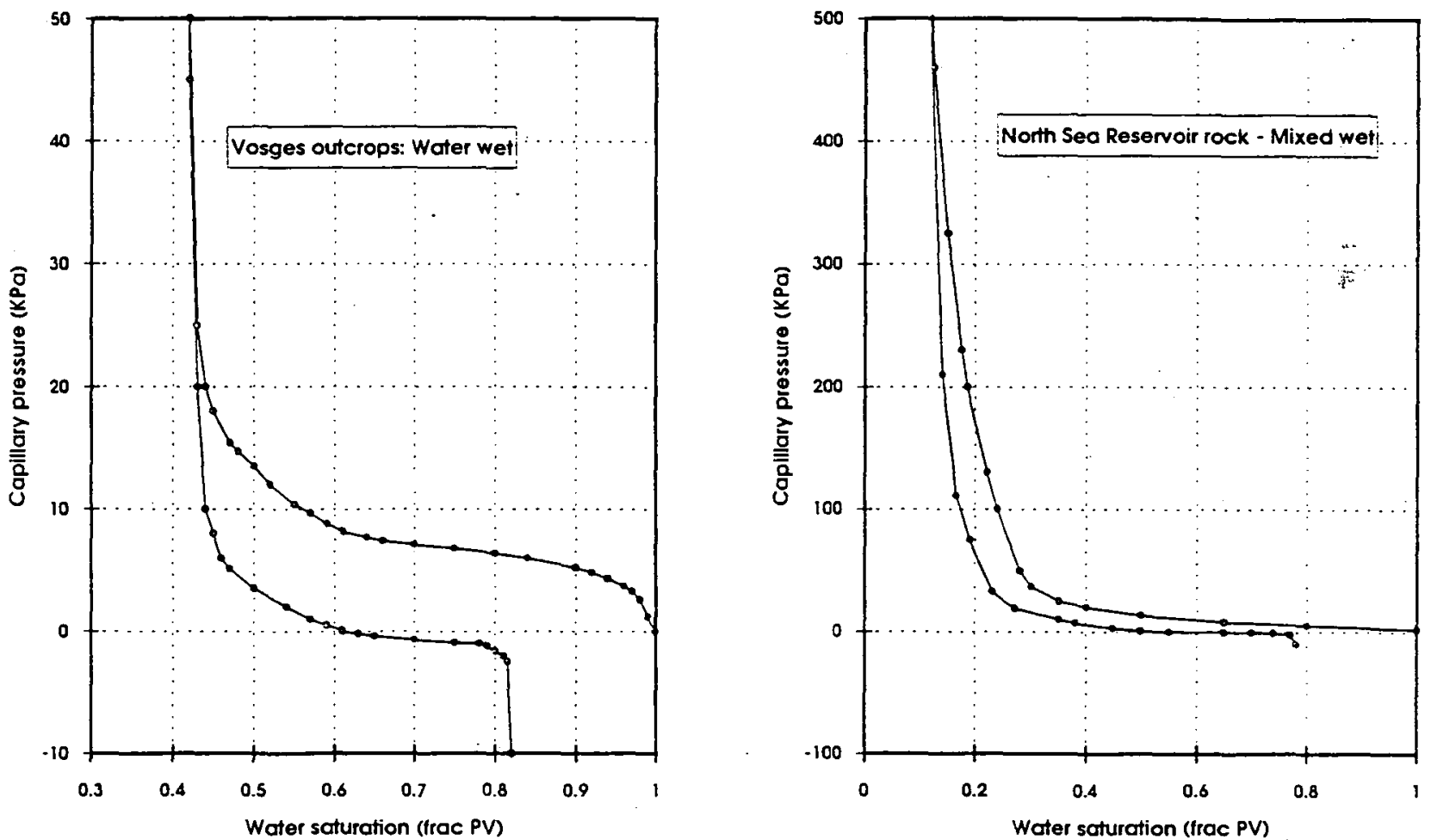

Figure 2 - Capillary pressure curves: a) Water wet core; b) Mixed wet core.

Simulations have been performed with emphasis on the initial water saturation profiles established prior to a waterflooding experiment. Two sets of rock characteristics were used: Strong water-wet Volges outcrop and a mixed wet rock from a North Sea reservoir. Table 1 summarises the basic core and fluid data used. Figure 1 and 2 depict the relative 


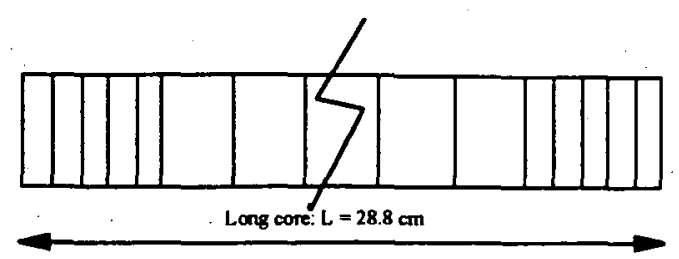

Figure 3: 1D grid system with grid refinement at core inlet and outlet.

permeability and capillary pressure loops for the two rock types studied.

1D grid system has been applied. The total number of numerical blocks is 50 for the short core case (5 $\mathrm{cm})$ and 60 for the long core case $(28.8 \mathrm{~cm})$ with grid. refinement at the core inlet and outlet. as shown in Figure 3. Numerical dispersion is assumed to be small compared to the effects studied.

Two methods for establishing initial water saturation have been simulated: i) Centrifuge drainage; and ii) High rate oil flooding. The simulations started with $100 \%$ water saturated core. Water was drained from the core with high speed centrifugation or high oil flooding. Afterwards, a sufficient shut-in time was simulated to allow water redistribution to happen. Then, simulation of a normal waterflood was performed with the purpose of determining relative permeability curves. The rate applied was considered to be high enough to ignore capillary effect. JBN-method is then used to convert the simulated production and pressure data to the desired relative permeability curves. The initial water saturation in the JBN-interpretation is the average water saturation at the end of oilflooding. Table 2 gives the sequences of the events simulated.

\section{Simulation results and discussions}

Figures 4,5 and 6 show the water saturations at the end of each event. The water saturation at the end of the drainage is highly non-uniform characterised by the water saturation buildup at the outlet end due to the capillary end-effect. After the core was released from centrifugation or oil flood, the water saturation redistribution occurred. At this stage, water saturation decreases at one part of the core where water saturation is high (outlet). while increases at another part where water saturation is low. The water saturation reaches a capillary equilibrium state which is controlled by the relative permeability and capillary pressure scanning curves. As the saturation history is different for different location of the core. the final water saturation is slightly non-uniform. The core is then undergone a waterflooding for determining relative permeability curves by JBN-method. The final relative permeability curves calculated from the simulated responses are plotted together with the "true" curves for comparison in Figures 1 and 2.

\section{Table 1 - Core and fluid data}

\begin{tabular}{lc}
\hline Water wet core & $:$ \\
Core length $(\mathrm{cm})$ & 28.8 \\
Core cross section $\left(\mathrm{cm}^{2}\right)$ & 10.64 \\
Porosity $(\%)$ & 22.0 \\
$\mathrm{Kro}(\mathrm{Swi})(\mathrm{mD})$ & 500 \\
$\mathrm{~S}_{\mathrm{mi}}(\%)$ & 42.0 \\
$\mathrm{~S}_{\text {or }}(\%)$ & 18.0 \\
& \\
Mixed wet core & Short /long \\
Core length $(\mathrm{cm})$ & $5.0 / 28.8$ \\
Core cross section $\left(\mathrm{cm}^{3}\right)$ & $11.40 / 10.64$ \\
Porosity $(\%)$ & $20.0 / 22.0$ \\
Kro(Swi) $(\mathrm{mD})$ & $500 / 2000$ \\
$\mathrm{~S}_{\text {wi }}(\%)$ & 11.0 \\
$\mathrm{~S}_{\text {or }}(\%)$ & 25.0 \\
& \\
Fluid data & \\
Oil viscosity $(\mathrm{cp})$ & 1.06 \\
Water viscosity $(\mathrm{cp})$ & 1.30 \\
Oil density $\left(\mathrm{g} / \mathrm{cm}^{3}\right)$ & 0.780 \\
Water density $\left(\mathrm{g} / \mathrm{cm}^{3}\right)$ & 1.012 \\
\hline \hline
\end{tabular}

Table 2 - Data for simulation events Centrifuge experiment for Swi estab. (mixed wet)

Centrifugation speed (RPM) 4000

Distance from rotation axis to the core center $(\mathrm{cm})$

Centrifugation time (hours)

Shut-in times (hours)

\section{Flooding experiment 1 (water wet) \&} 2 (mixed wet core)

Oil flooding rate (cc/min)

Rate 1

Rate 2

Shut-in (hours)

Water flooding rate (cc/min) 


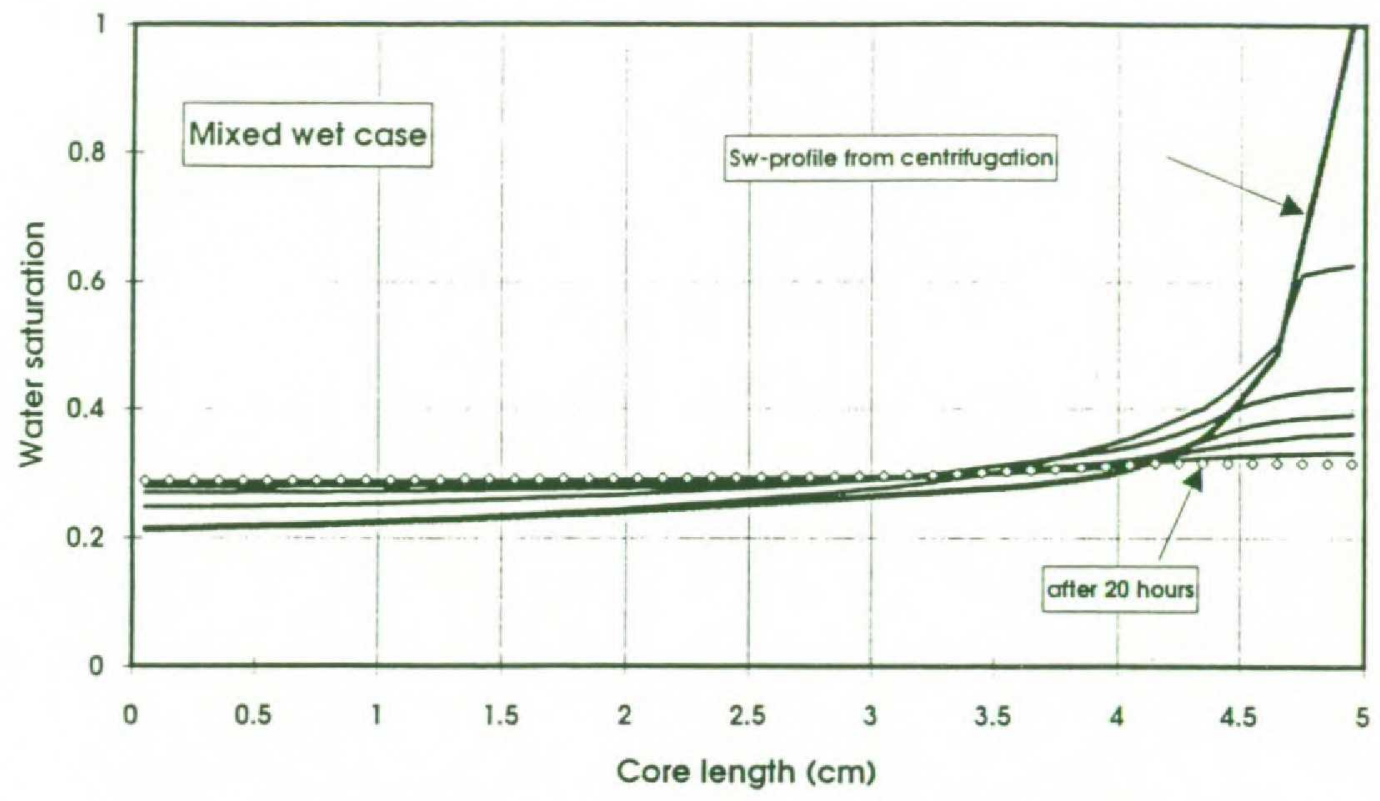

Figure 4 - Water saturation distribution after centrifugation.

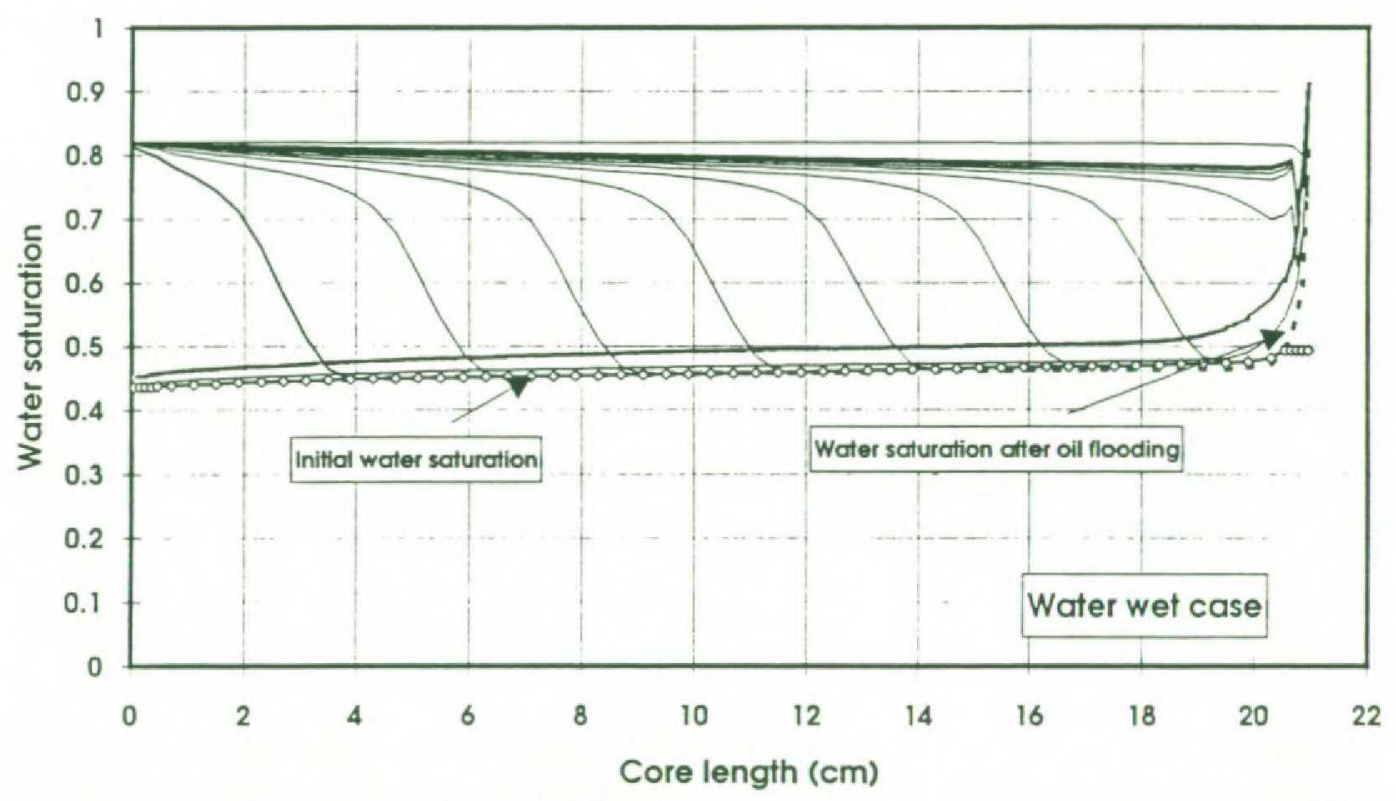

Figure 5 - Water saturation distribution at different stage of flooding (Water wet)

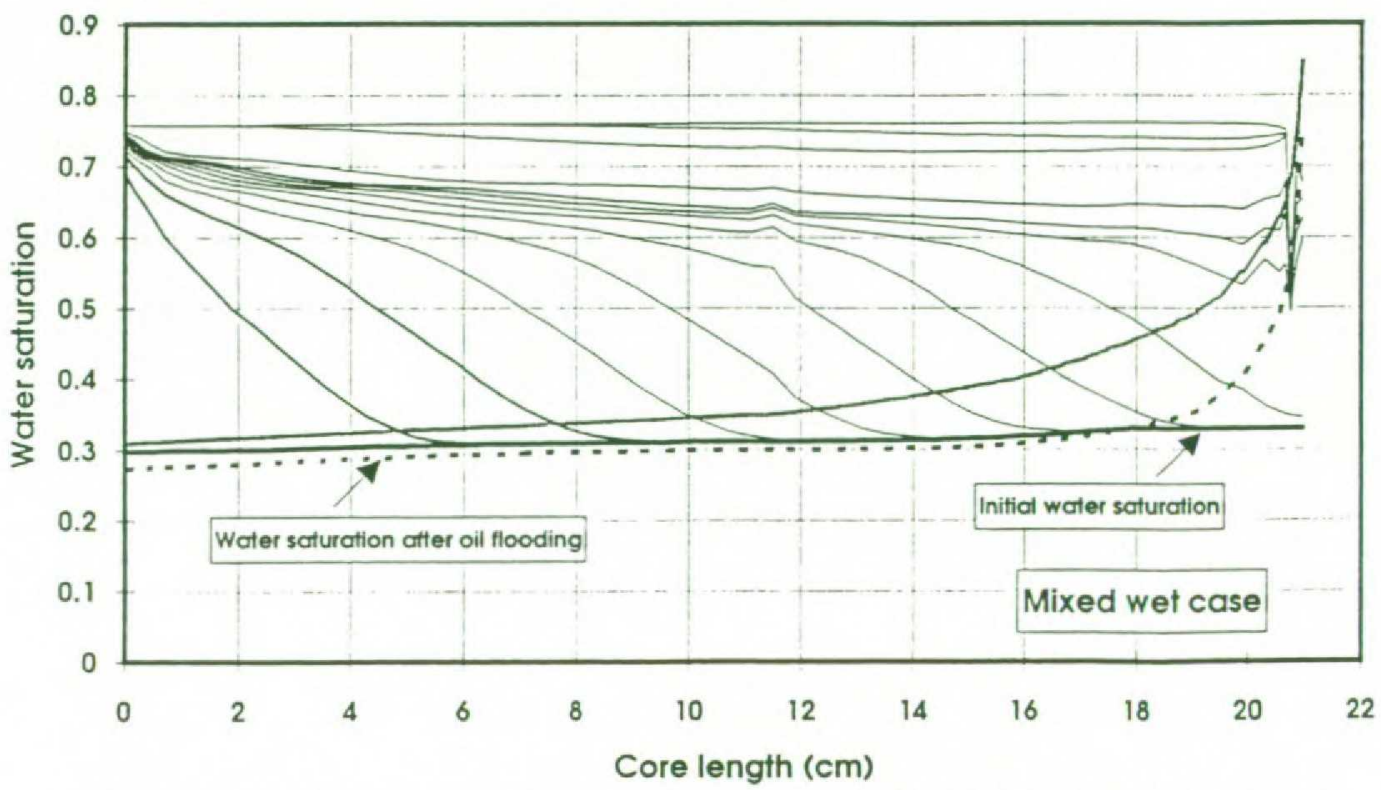

Figure 6 - Water saturation distribution at different stage of flooding (Mixed wet) 


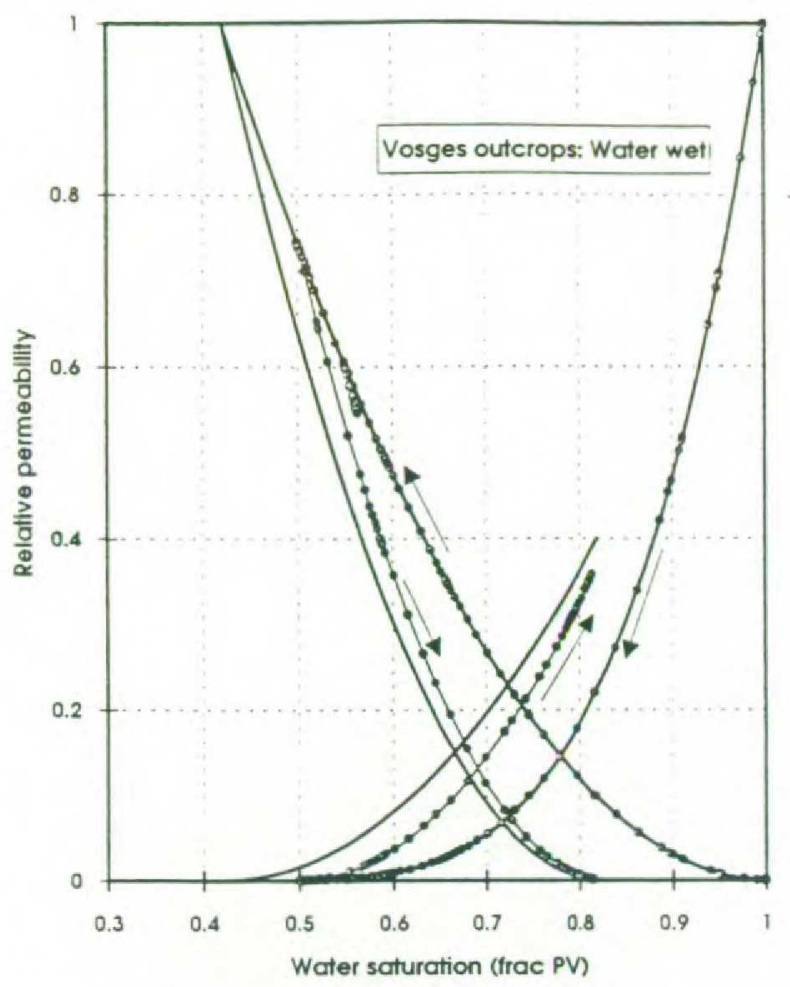

Figure 7 - Relative permeability curves at core outlet.

As can be seen, the rel. perm. curves obtained by JBN-method is more or less a scanning curve rather than the bounding rel. perm. curves, especially for the mixed wet case In addition, the different saturation history at different location of the core can be transplanted to the residual oil saturation, which is, according to Land's formula. dependent on the historically maximum oil saturation. This effect has not been studied in detail.

The water saturation profile at the start of waterflooding can be characterised as slightly non-uniform, as can be seen in Figure 5 and 6 . The saturation difference at inlet and outlet is less than $5 \%$ pore olume. However, because of the difference in saturation history, with resulting hysteresis effect, the relative permeability and capillary pressure values are significantly deviated from the bounding curves. Figure 7 and 8 show the scanning relative permeability and capillary pressure curves assigned to the core outlet for the all simulated events. Even for the water wet case, the rel.perm. at the core outlet departs from the bounding curve with significant distance. However, for this case, the portion of the core affected by the hysteresis is relatively small, and the overall average of the interpreted relative permeability

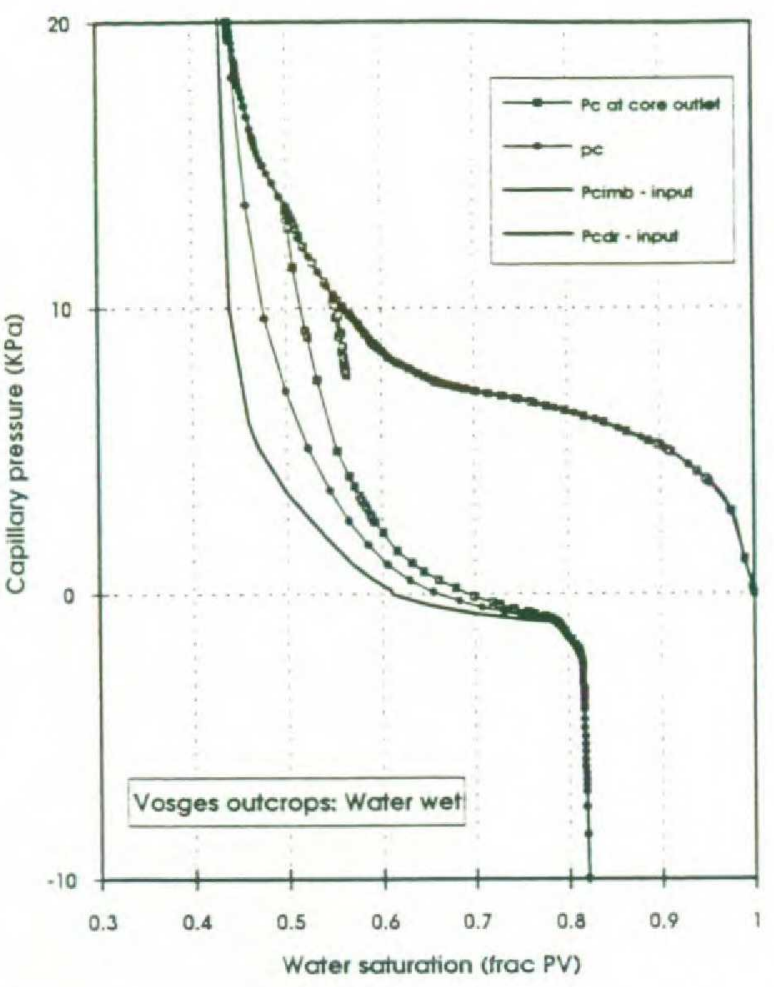

Figure 8 - Capillary pressure curve at core outlet.

curves seem to be satisfactory. For the mixed wet core. the effect is too obvious to be ignored.

Besides the hysteresis effect. the capillary pressure itself and its effect on the interpretation also is a issue for discussion. In a waterflooding experiment. the final water saturation often is not the true residual oil saturation due to capillary trapping, specially for mixed-wet cases. When this saturation is interpreted as the residual oil saturation with corresponding oil relative permeability equals to zero, the oil rel.perm. will be underestimated. The rel.perm. values from the JBN-interpretation in Figures 1 and 2 might contain this effect in addition to the influence from hysteresis phenomenon. It is therefore important to interpret relative permeability curves from waterflooding experiments incorporated with capillary pressure information. Numerical simulations seem to be a unique and effective tool for improved interpretation and quality control.

\section{Conclusions}

The commonly used laboratory procedure for establishing initial water saturation prior to a waterflood experiment for relative permeability measurement can give a non-uniform initial water 
profile. Although redistribution of it aided by capillary pressure helps to give a fairly uniform initial water saturation, the hysteresis effect described by scanning curves can be significant to affect the finally derived relative permeability curves.

Diaphragm method should be considered at the best method for initial water saturation establishment whenever possible. Centrifuge and oilflooding method may be subjected to hysteresis effect, and must be used with caution. The final results should be checked with numerical simulations with hysteresis option.

When the water saturation is subjected to different saturation history at different location of the core, the resulting relative permeability curves are deviated from the bounding curves. Mixed wet core are more affected than strongly water-wet cores.

\section{References}

1. Longeron. D., Hammervold W.L. and Skjaveland, S.M.: "Water-Oil Capillary Pressure and Wettability Measurements Using Micropore Membrane Technique", paper presented at the 1994 International Symposium of the Society of Core Analysts. Stavanger, Sept.12-14.

2. Guo. Y. and Hammervold, W.L.: "Equilibration Time and Accuracy of Capillary Pressure Measure Using Diaphragm Method", paper presented at 34th Annual SPWLA Symposium and the 14th Formation Evaluation Symposium, June 13-16. 1993. Calgary, Canada.

3. Ying Guo, Jan-Erik Nordtvedt and Henning Olsen: "CENDRA+ User's Manual. version 2.3/3.3", RF-Rogaland Research report No. 183/93, 1993.

4. J.E. Killough: "Reservoir Simulation With History-Dependent Saturation Functions". SPEJ. February 1976.

\section{Appendix A: Implementation of relativie permeability and capillary hysteresis models:}

Killough's hysteresis models ${ }^{4}$ for relative permeability and capillary pressure scanning curves have been implemented in CENDRA with slightly modification. The saturation history is divided into two categories (Loop 1 and Loop 2) and four types, as shown in Figure A. The four types (referred to as 1 to 4) are defined as dependent the saturation history. They are:

1. Primary drainage: Water saturation initially equals to 1.0 , and decreases.

2. Primary imbibition: Water saturation initially equals to $S_{w i}$, and increases.

3. n-th drainage: Water saturation decreases after i) a primary imbibition; or ii) successively after a n-th imbibition

4. n-th imbibition: Water saturation increases after i) a primary drainage; or ii) successively after a $\mathrm{n}$-th drainage.

Land's formulation for prediction of the historydependent residual non-wetting phase. Although this is only valid for strongly non-wetting phase, no other general model is available for this purpose. Land's equation is expressed as

$$
S_{o r}^{\text {hyst }}=\frac{1-S_{w}^{\text {hyst }}}{1+c \cdot\left(1-S_{w}^{\text {hyst }}\right)} \quad, \quad c=\frac{1}{S_{o r}^{\max }}-\frac{1}{S_{o}^{\max }}
$$

where $S_{w}^{h y s t}$ is updated when $S_{w}$ changes its direction, and $S_{o r}^{\text {hyst }}$ is updated when its previous value is exceeded at the above event. The new end-point for imbibition scanning curve is calculated from

$$
\begin{aligned}
& K_{r w}^{i m}\left(S_{o r}^{h y s t}\right)=K_{r w}^{d r}\left(S_{o r}^{h y s t}\right)+ \\
& {\left[K_{r w}^{i m}\left(S_{o r}^{h y s t}\right)-K_{r w}^{d r}\left(S_{o r}^{\max }\right)\right] \cdot\left(\frac{S_{o r}^{h y s t}}{S_{o r}^{\max }}\right)^{a}}
\end{aligned}
$$

where the parameter a defines the curvature of the locus of the maximum for the end-point water imbibition curve. In the present version of CENDRA, $a$ is set to be 1.0.

A normalised water saturation is used in the equations for the scanning curves: 


\section{Definition of hysteresis path}
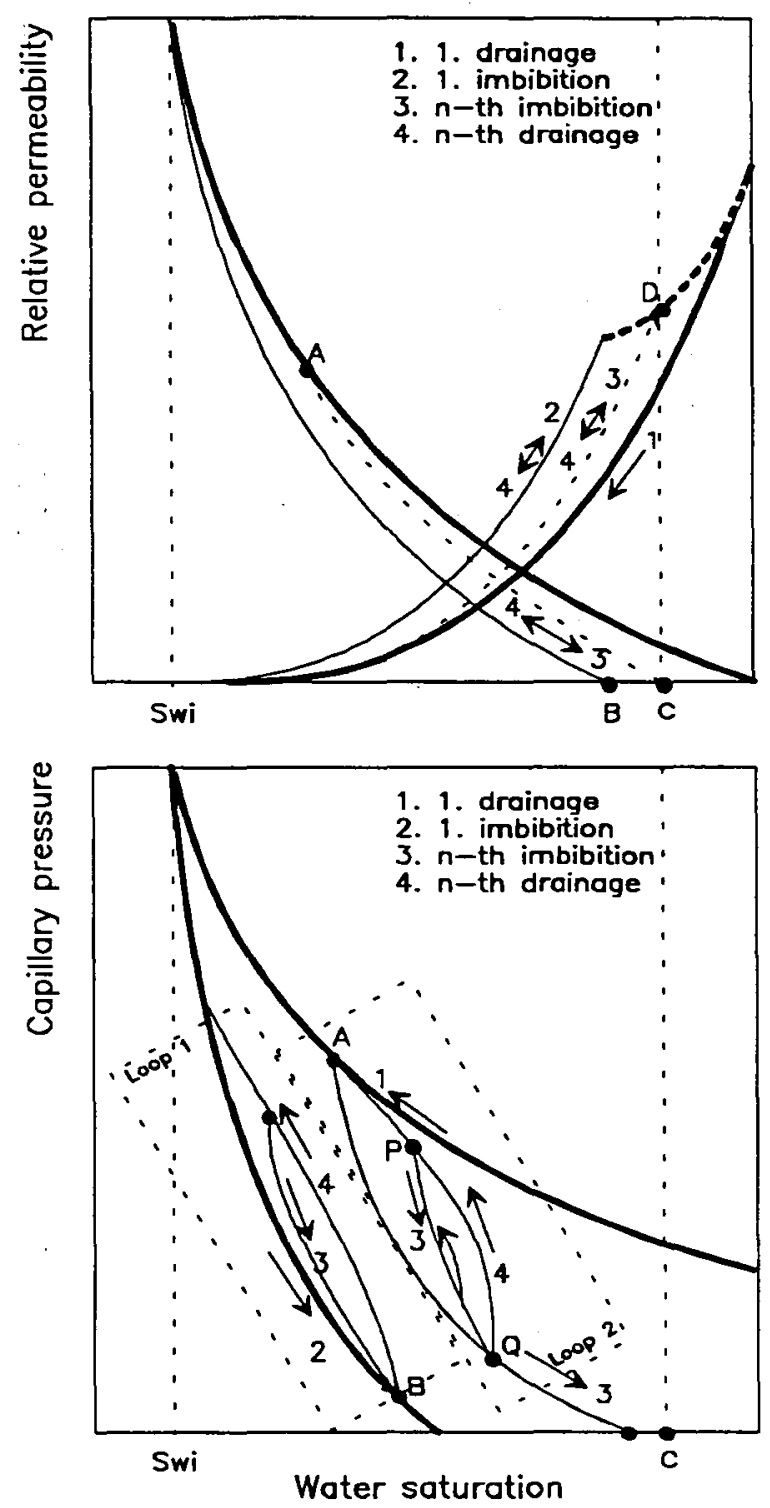

Figure A - Definition of hysteresis processes for relative permeability and capillary pressure curves.

$S_{w}^{n o r m}=S_{o r}^{\max }+\left(1-S_{w}-S_{o r}^{\text {hyst }}\right) \frac{S_{o}^{\max }-S_{o r}^{\max }}{1-S_{w}^{\text {hyst }}-S_{o r}^{\max }}$

For the drainage to imbibition process, the scanning curves are interpolated from $S_{w}^{\text {hyst }}$ to $S_{o r}^{\text {hyst }}$ by using the experimental imbibition and drainage curves.

$$
\begin{aligned}
K_{r w}^{s c a n}= & K_{r w}^{d r}\left(S_{w}^{h y s t}\right)+ \\
& {\left[K_{r w}^{i m}\left(S_{o r}^{h y s t}\right)-K_{r w}^{d r}\left(S_{w}^{h y s t}\right)\right] } \\
& \frac{K_{r w}^{i m}\left(S_{o r}^{n o r m}\right)-K_{r w}^{i m}\left(S_{o}^{\max }\right)}{K_{r w}^{i m}\left(S_{o r}^{\max }\right)-K_{r w}^{i m}\left(S_{o}^{\max }\right)} \\
K_{r o}^{\text {scan }}= & K_{r o}^{d}\left(S_{w}^{\text {hyst }}\right) \cdot \frac{K_{r o}^{i m}\left(S_{o r}^{n o r m}\right)-K_{r o}^{i m}\left(S_{o}^{\max }\right)}{K_{r o}^{i m}\left(S_{o r}^{\max }\right)-K_{r o}^{i m}\left(S_{o}^{\max }\right)}
\end{aligned}
$$

For the capillary scanning curves, the scanning curves originated from Loop 1 are different from those originated from Loop 2. As assumption, if the saturation changes direction at a scanning curve (e.g. path 3 from point P) the scanning curves are formulated such that the target of the scanning curve is the latest turn-around point (e.g. point Q).

The equations used for generating scanning curves are:

Drainage scanning curve from bounding imbibition curve (loop 1 - type 2 to 4):

$$
\begin{aligned}
P_{c d r}^{s c a n}=P_{c}^{i m}\left(S_{w}\right)+ & {\left[\frac{\frac{1}{S_{w}^{h y s t}-S_{w}+\varepsilon}-\frac{1}{\varepsilon}}{\left.\frac{1}{S_{w}^{h y s t}-S_{w r}+\varepsilon}-\frac{1}{\varepsilon}\right]}\right.} \\
& {\left[P_{c}^{d r}\left(S_{w}\right)-P_{c}^{i m}\left(S_{w}\right)\right] }
\end{aligned}
$$

Imbibition scanning curve from bounding drainage curve (loop 2 - type 1 to 3):

$$
\begin{aligned}
P_{c i m}^{s c a n}=P_{c}^{d r}\left(S_{w}\right)- & {\left[\frac{\frac{1}{S_{w}-S_{w}^{\text {hyst }}+\varepsilon}-\frac{1}{\varepsilon}}{\frac{1}{S_{w}^{\text {max }}-S_{w}^{\text {hyst }}+\varepsilon}-\frac{1}{\varepsilon}}\right] } \\
& {\left[P_{c}^{d r}\left(S_{w}\right)-P_{c}^{i m}\left(S_{w}\right)\right] }
\end{aligned}
$$

n-th turn-around drainage scanning curve (type 3 to 4) 


$$
\begin{aligned}
P_{c d r}^{s c a n}=P_{c}^{i m}\left(S_{w}\right)+ & {\left[\frac{\frac{1}{\Delta S_{w}^{h y s t}+\varepsilon}-\frac{1}{\varepsilon}}{\frac{1}{\Delta S_{w}+\varepsilon}-\frac{1}{\varepsilon}}\right] } \\
& \cdot\left[P_{c}^{d r}\left(S_{w}\right)-P_{c}^{i m}\left(S_{w}\right)\right] \\
& +\delta P_{1}-\delta P_{2}
\end{aligned}
$$

n-th turn-around imbibition scanning curve (type 4 to 3)

$$
\begin{aligned}
P_{c i m}^{s c a n}=P_{c}^{d r}\left(S_{w}\right)- & {\left[\frac{\frac{1}{\Delta S_{w}^{h y s t}+\varepsilon}-\frac{1}{\varepsilon}}{\frac{1}{\Delta S_{w}+\varepsilon}-\frac{1}{\varepsilon}}\right] } \\
& \cdot\left[P_{c}^{d r}\left(S_{w}\right)-P_{c}^{i m}\left(S_{w}\right)\right] \\
& +\delta P_{1}-\delta P_{2}
\end{aligned}
$$

The saturation ranges $\Delta S_{w}^{\text {hyst }}, \Delta S_{w}, \delta P_{1}$ and $\delta P_{2}$ are defined according to the saturation history:

$S_{w 1} \quad$ Turn around saturation from imbibition to drainage

$\mathrm{S}_{\mathrm{w} 2} \quad$ Previous turn around saturation from drainage to imbibition

$P_{c}^{s c}\left(S_{w 1}\right) \quad$ Capillary pressure on the scanning curve $S_{w 1}$

$P_{c}^{s c}\left(S_{w 2}\right) \quad$ Capillary pressure on the scanning curve at $S_{\mathrm{w} 2}\left(S_{\mathrm{w} 2}<S_{\mathrm{w} 1}\right)$

$$
\begin{aligned}
& \Delta S_{w 1}^{h y s t}=S_{w 1}-S_{w} \\
& \Delta S_{w 2}^{h y s t}=S_{w 1}-S_{w 2} \\
& \Delta S_{w}=S_{w 1}-S_{w 2} \\
& \delta P_{1}=\left[P_{c}^{s c}\left(S_{w 1}\right)-P_{c}^{i m}\left(S_{w 1}\right)\right] \frac{\Delta S_{w 2}^{h y s t}}{\Delta S_{w}} \\
& \delta P_{2}=\left[P_{c}^{d r}\left(S_{w 2}\right)-P_{c}^{s c}\left(S_{w 2}\right)\right] \frac{\Delta S_{w 1}^{h y s t}}{\Delta S_{w}}
\end{aligned}
$$

